\title{
Effect of Functional Endoscopic Sinus Surgery on Voice and Speech Recognition
}

\author{
Estefanía Hernández-García, ' Laureano Moro-Velázquez, Ramón González-Herranz, \\ Juan Ignacio Godino-Llorente, and Guillermo Plaza,
}

\begin{abstract}
Summary: Objective. Functional Endoscopic Sinus Surgery (FESS) is the surgery of choice for nasal polyposis and chronic rhinosinusitis. The aim of our study is to assess the influence of this surgery in the acoustic parameters of voice, and their implications in the systems of identification or verification of the speaker through the speech.

Material and methods. A prospective study was performed between January 2017 and June 2017 including two groups of patients: those undergoing FESS, and a control group. Demographic data and GRBAS assessment were statistically analyzed. In addition, a recording of patients' voices was made with a subsequent acoustic analysis and automatic identification of the speaker through machine learning systems, establishing the equal error rate. Samples were taken before surgery, 2 weeks after surgery and 3 months later.

Results. After FESS, a significant difference was observed in Grade, Roughness, Breathiness, Asthenia, Strain (GRBAS). Besides, acoustic analysis showed a significance decrease in fundamental frequency (F0), when compared with the control group. For the automatic identification of the speaker through computer systems, we found that the equal error rate is higher in the FESS group.

Conclusions. Results suggest that FESS produce a decrease of F0 and changes in the vocal tract that derive in an increase in the error of recognition of the speaker in FESS patients.
\end{abstract}

Key Words: FESS-Acoustic analysis-Formants-Automatic identification.

\section{INTRODUCTION}

The vocal tract includes various structures that begin from the vocal folds passing through the oropharynx and the nasal cavity ending at the lips and nostrils. Hence, theoretically, changes in any of these structures may affect the acoustic properties of the radiated sound signal also changing the response of speech recognition systems to the speech of the patient.

Polyposis is a disease of the nasal mucosa that leads to an occupation of the nasal passages and paranasal sinuses. ${ }^{1}$ This occupation produces a reduction of the passage of air through the nostrils, disturbing the voice and producing the nasalization, so called closed rhinolalia.

Regarding treatment, the first step indicated is medical treatment, using topical corticosteroids as the first line, and other treatments such as antileukotrienes or oral steroids. ${ }^{1}$ When intensive medical treatment is not effective, the next step is surgery.

Functional endoscopic sinus surgery (FESS) is a very frequent procedure for the correction of nasal polyposis and chronic rhinosinusitis. ${ }^{2}$ This has been the preferred technique during the last 30 years in contrast to external nasal surgery, with the improvement of optics, visualization systems, and less aggressive approaches. ${ }^{3}$

Employing FESS, the nasal inflammatory mucosa and bone ethmoid cells are eliminated, improving significantly the passage of air through the nose and decreasing rinolalia. In addition, this opening of the paranasal sinuses toward the fossa and changes in the nasal cavity has an impact in the acoustic resonance and could alter the patient's perception of their own speech. However, several authors have studied these changes, ${ }^{4-9}$ without reaching clear conclusions.

On the other hand, the systems of identification or verification of the speaker through the speech (SISS) have emerged thanks to the use of machine learning technologies, allowing to recognize speakers, through the use of computational systems in different platforms such as mobile phones. ${ }^{10,11}$ Today, there are many sensitive or personal data in our computing devices that we want to keep private. Limiting the access to them through identification systems might be crucial. Also, the use of these technologies is common in bank transactions made by phone or other remote activities in which the identity of the speaker must be verified.

A large number of acoustic features have been employed in SISS schemes. In the literature, the most used speech signal characterization coefficients are Mel Frequency Cepstrum Coefficients (MFCC), ${ }^{12}$ that combined with machine learning classification techniques allow to automatically identify the speaker. These features contain information about the vocal tract during the production of speech and are widely used in not only for identification or verification of the user but with other purposes such as speech or language recognition.

The aim of this study is to evaluate changes in the acoustic analysis in a group of patients before and after FESS, 
and the impact of this surgery in speaker recognition systems. This will allow to evaluate the impact of the surgery on the quality of the voice, the perceptual assessment, and the acoustic characterization of the speech signal.

\section{MATERIALS AND METHODS}

A prospective study was performed between January 2017 and June 2017 including patients operated on FESS and a control group, at a University Hospital. This study was approved by the ethics committee of the hospital and all participants signed informed consent.

\section{Database}

Two groups of subjects were defined: those having nasal obstruction with polyposis and undergoing FESS (FESS group), and a control group without nasal obstruction (control group). The FESS group included patients to which the medical treatment failed and therefore needed a surgical solution. The control group included patients from the surgical waiting list for some minor intervention under local anesthesia that did not affect the upper airway not the vocal folds.

The inclusion of patients in the FESS group was prospective, fulfilling the following criteria of inclusion: age over 18 years, with symptoms of nasal obstruction and diagnosis of polyposis (more than grade 2 in Lildholdt scale) by nasal endoscopy, and severe extension of polyposis as confirmed by CT scan, showing Lund-Mackay scores higher than 10 in all cases. Several scales can be used for classification, including classification of Lildholdt, being one of the best methods for its assessment. It varies from 0 (absence of polyps) to 3 (massive polyposis). On the other hand, the scale of Lund-Mackay is a radiological scale used for the classification of sinus involvement there have been different proposals throughout the years. This scale scores all the sinuses of each side as a function of its involvement and the osteomeatal complex, ranging from 0 to 24 considering both nostrils, and from 0 to 12 for each nostril. For all the referred patients, FESS was performed by the same surgeon under general anesthesia.

Exclusion criteria were any type of organic or neurological pathology related to phonation or speech or having undergone any previous otorhinolaryngological surgical procedure; patients with pathological GRBAS (more than four) or with evident pathology in the vocal folds observed in the fibroscopy were also excluded from the study.

Our protocol included the observation of demographic data (age, gender) main symptoms, weight and height, tobacco use, and the professional use of their voice (ie, whether they have or not an intensive use of the voice in their professional activity).

All patients were evaluated at baseline (session 1), 2 weeks after surgery (session 2), and 3 months later (session 3 ).

\section{GRBAS and acoustic parameters}

At each session, a GRBAS evaluation ${ }^{13}$ was also performed as a subjective assessment of the quality of the patient's voice. as done in other similar studies. ${ }^{714}$ Patients were asked at every session if they had perceived differences in their voice after the surgery, in order to register the subjective self-evaluation of the patient in our database.

Finally, voice and speech recordings were made in a soundproofing and acoustically conditioned room, employing an AKG C420 headset microphone, and a Soundblaster Live 24 bits sound card connected to a personal computer equipped with the PRAAT software. The recording protocol was the same in the three sessions, collecting three utterances of the vowel /a/ with a duration of at least 3 seconds, along with the running speech corresponding to the description of an illustration given to the patient. Besides, the 5 Spanish vowels / a /, / e /, / i /, / o / and / u / were recorded once, although only the three repetitions of / a / was analyzed as this vowel is the most similar to the phoneme / ae / in English and is the most used in literature.

The voice and speech were recorded at comfortable pitch and loudness.

The acoustic analysis of the vowel /a/ was made using the tools provided in the WPCVox package and using ad hoc algorithms developed to run in a Matlab environment. WPCVox ${ }^{15}$ is an acoustic analysis software oriented to the voice quality measurement in clinical environments, professional voice, and scientific research. The following parameters were extracted using this software: fundamental frequency (F0), Jitter, Shimmer, Harmonic to Noise Ratio (HNR), and Noise to Harmonic Ratio (NHR). Additionally, a Matlab-based software has been used to obtain different parameters related to the resonance of the vocal tract, following the algorithms described by Mehta et al in $2012^{16}$; for F0, Jitter, Shimmer, HNR, and NHR. The obtained features were averaged using the three repetitions of the vowel /a/ to avoid the selection bias.

All patients underwent the Voice Handicap Index (VHI) test before surgery, if there was a high VHI they were excluded from the study. Later, it was carried out in the same visits, since there was no difference and a homogeneous behavior, it was decided not to include it in the study.

\section{Methodology of SISS}

The SISS are based on complex engineering systems with different types of acoustic features and classification systems. In our study, the MFCC characterization of the speech was employed along with an i-vector-Probabilistic Linear Discriminant Analysis (PLDA) ${ }^{17}$ classification system in order to identify the different speakers from both groups using their speech.

For this purpose, the KALDI framework ${ }^{18}$ was used, trained with those utterances of running speech recorded during the description of an image. The following procedure is followed to automatically identify the speaker:

- Phase 1: the speech is normalized and resampled at 8 $\mathrm{kHz}$; then, a framing of the acoustic signal is performed, obtaining speech segments of 25 milliseconds 
length every 10 milliseconds, shaped using Hanning windows. The speech from the three different sessions is treated separately.

- Phase 2: each of these segments of speech is parameterized, yielding a series of vectors (one vector for each frame) with a number of $20 \mathrm{MFCC}$, characterizing the signal. First and second derivatives of the coefficients are added to include information of the speed and acceleration of variation. Thus, each frame is characterized by a vector of 60 coefficients.

- Phase 3: silences are removed, rejecting the vectors of characteristics belonging to the instants in which there is no speech.

- Phase 4: the training of a model per speaker is carried out. The method requires adjusting an initial model by adapting a generic statistical model called Universal Background Model, which was initially obtained using the Albayzin database ${ }^{18}$ (resampled at $8 \mathrm{kHz}$ to match the same conditions as the corpus used in this study). The Universal Background Model is adapted to the database recorded in this work to obtain a new statistical model calculated using the acoustic material belonging to the first session (before surgery). This new statistical model is the basis to obtain the i-vector which uniquely characterizes each speaker belonging to session 1 .

- Phase 5: the identification is performed following an ivector-PLDA classification. Once the i-vector of each speaker has been obtained, this is used to compare among speakers and different sessions through PLDA providing a score, which is directly linked with the probability that the utterance belongs to a speaker or not (ie, it provides an objective measure about how close a new i-vector is to the trained $\mathrm{i}$-vector corresponding to a certain speaker). Thus, the overall scheme includes one i-vector per speaker (before surgery), which is used to obtain different scores by comparison with the i-vectors obtained from every speaker and testing utterance of the three sessions available. Roughly speaking, the trained i-vector which minimizes the score for a given utterance is supposed to be the one of the speaker the acoustic material belongs to.

- Phase 6: the scores given by all the speakers are analyzed to obtain the Equal Error Rate (EER) of session 1 , session 2, and session 3. The EER is the point in which false acceptance ratio is equal to the false rejection ratio of the system. Error rates are defined as the ratio between the number of errors produced by the system and the errors made during the comparison between reference patterns and test characteristics.

We use a scheme based on MFCC and i-vectors since this is the most accepted scheme in the state of the art for automatic speaker recognition and verification purposes. ${ }^{19}$

In this study, EER is used to measure the identification capabilities of the analyzed SISS. EER is one of the most popular figures of merit in the automatic identification-verification of speaker's systems. It is defined at the point in the score axis where the false acceptance rate and the false rejection rate are equal. The higher the EER, the higher the error in identifying that speaker.

Thus, in this study, the scores obtained with the speakers of session 1 are employed to obtain the EER 1; the scores from session 2 allow to obtain EER; and those from session 3, EER3. The comparison of the different EER in the two speaker groups (FESS and controls) allows to determine if the speaker features are different enough to produce changes in the identification behavior of the classifier.

\section{Statistical analysis}

An analysis of the calculated acoustic parameters and those measured for the three sessions was carried out, with a statistical analysis study performed with STATA 11.1 software. The GRBAS index was measured as a continuous quantitative variable in Table 1 for the presurgical comparison between groups, but then analyzed as a categorical variable (adding the total of the values in each GRBAS category and giving a value that in the series ranged from 0 to 3, at most). We applied Wilcoxon paired test to quantitative variable with no normal distribution intragroup, Wilcoxon's test between groups, or Fisher's exact test for categorical variables. Hence, we used ANOVA for variables with homogeneous variances and Kruskal Walliss test for variables with heterogeneous variances. Holm-Bonferroni correction was used when multiple repeated measures were done. The $P$ value was calculated by Fisher's Exact Test.

\section{RESULTS}

A total of 67 patients distributed in two groups were assessed: those operated on FESS; and the control group. Throughout the study, a total of 14 patients were not included (nine in the control group and five in the FESS group), since some of them abandoned or due to problems in the recording of some sessions, mainly sound interferences that invalidated the data. Finally, a total of 53 patients had all visits and recordings available to be evaluated, 26 in FESS group and 27 in the control group. The demographic data are shown in Table 1. No significant differences were observed between both groups in gender or age.

Baseline data showed significant differences in GRBAS between both groups (2.15 media in FESS group versus 0.63 media in control group). The data obtained comparing the two groups in the three sessions (baseline, 2 weeks and 3 months), concerning GRBAS are shown in Table 2.

When patients were questioned on their perception of their voice after the surgery, 50\% of them had appreciated changes in their voice (Table 1). This change was always considered as positive by patients, who observed an improvement of their nasality and without noticing differences in effort or vocal fatigue to perform the phonation. In the other $50 \%$ who did not notice susceptible changes, VHI was similar to the previous ones.

The private use of SISS by patients with their own electronic recognition systems was not registered. 
TABLE 1.

Demographic Data of Patients and Baseline Nasalance Differences Between Both Groups

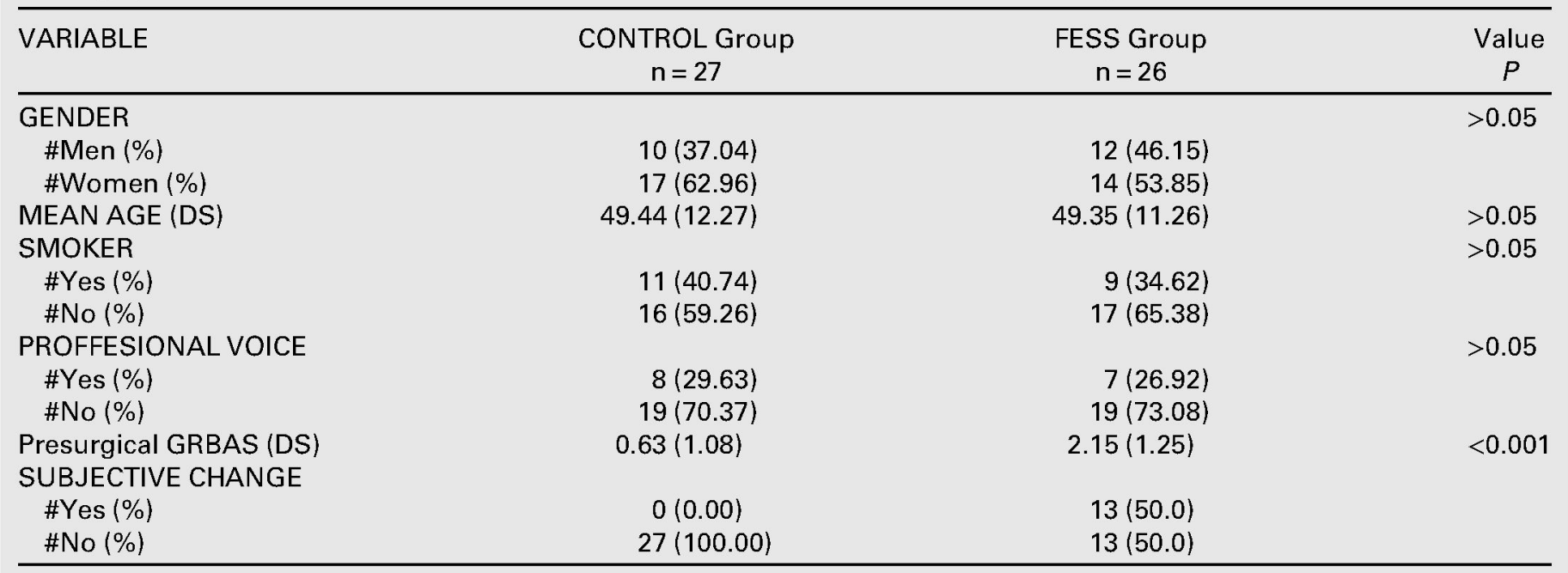

With reference to the acoustic analysis, the main results are presented in Table 3. To reduce the inherent intraspeaker variability, the statistical analysis was carried out after averaging the acoustic parameters obtained from the three utterances of the sustained /a/ vowel recorded in each session. Neither HNR, NHR, Jitter, nor Shimmer varied in any cohort during the study. Nevertheless, 3 months later, F0 significantly decreased in FESS group. The ANOVA and Holm-Bonferroni did not find any heterogeneous variables or cofactors influence.

On the other hand, the EER analysis of the SISS is showed in Table 4. In each of the cases, the scores of each of the speakers with respect to all the trained models (or ivectors) were used to calculate the EER of the global system. The higher the EER, the greater the degree error of the system to identify a specific speaker. It is possible to see that the EER 3 is higher in the FESS group; thus, there was a higher error to identify FESS patients 3 months after surgery, in comparison to the control group.

In addition, a correlation between patients who noticed the greatest subjective change after surgery, those with higher changes in $\mathrm{F} 0$, and those that the SISS detected worse was found.

\section{DISCUSSION}

The changes in the vocal tract after surgery, and more specifically in the nasal cavity, can affect the quality of the

TABLE 2.

Comparison Intragroups of GRBAS. Number of Patients and Percentage of Each GRABS

\begin{tabular}{|c|c|c|c|c|}
\hline Variable & Baseline $^{*}$ & 2 Weeks Postop $^{\dagger}$ & 3 Months Postop ${ }^{\ddagger}$ & Value $P^{\S}$ \\
\hline \multicolumn{5}{|l|}{ FESS group $(n=26)$} \\
\hline GRBAS mean (DS) & $2.15(1.25)$ & $1.69(1.09)$ & $0.92(1.02)$ & $<0.05$ \\
\hline $1(\%)$ & $0(0.0)$ & $0(0.0)$ & $0(0.0)$ & \\
\hline $2(\%)$ & $16(61.54)$ & $18(69.23)$ & $12(46.15)$ & \\
\hline $3(\%)$ & $0(0.0)$ & $0(0.0)$ & $0(0.0)$ & \\
\hline GRBAS mean (DS) & $0.63(1.08)$ & $0.68(1.11)$ & $0.59(1.08)$ & $>0.05$ \\
\hline $0(\%)$ & $19(70.4)$ & $17(68)$ & $20(74.1)$ & $>0.05$ \\
\hline $1(\%)$ & $1(3.7)$ & $1(4)$ & $0(0.0)$ & \\
\hline $2(\%)$ & $6(22.2)$ & $6(24)$ & $6(22.2)$ & \\
\hline $3(\%)$ & $0(0.0)$ & $0(0.0)$ & $0(0.0)$ & \\
\hline $4(\%)$ & $1(3.7)$ & $1(4)$ & $1(3.7)$ & \\
\hline
\end{tabular}

* Presurgical Parameter (Baseline).

+ Two weeks Postsurgical Measurement.

F Three Months Postsurgical Measurement.

${ }^{\S}$ Value of $P$. 
TABLE 3.

Intragroups Comparison of Acoustic Parameters Averaging the Results of the three utterances of the vowel/a/. Values in Hertz $(\mathrm{Hz})$

\begin{tabular}{|c|c|c|c|c|c|}
\hline Variable & Baseline* & 2 Weeks Postop $^{\dagger}$ & Value $P^{\ddagger}$ & 3 Months Postop ${ }^{\S}$ & Value $P^{\ddagger}$ \\
\hline Fo & $145.64(42.80)$ & $145.13(41.95)$ & $>0.05$ & $139.70(37.54)$ & $<0.05$ \\
\hline Shimmer & $4.06(1.64)$ & $3.92(1.48)$ & $>0.05$ & $4.00(1.65)$ & $>0.05$ \\
\hline HNR & $13.49(3.35)$ & $14.08(2.76)$ & $>0.05$ & $13.30(2.94)$ & $>0.05$ \\
\hline NHR & $0.03(0.024)$ & $0.031(0.029)$ & $>0.05$ & $0.04(0.038)$ & $>0.05$ \\
\hline Jitter & $2.0869(2.25)$ & $1.7184(1.69)$ & $>0.05$ & $2.1132(1.23)$ & $>0.05$ \\
\hline Shimmer & $4.0354(1.88)$ & $3.9846(1.47)$ & $>0.05$ & $3.7367(1.32)$ & $>0.05$ \\
\hline HNR & $13.476(3.97)$ & $13.836(3.69)$ & $>0.05$ & $13.016(3.45)$ & $>0.05$ \\
\hline NHR & $0.0581(0.09)$ & $0.0383(0.04)$ & $>0.05$ & $0.0369(0.029)$ & $>0.05$ \\
\hline
\end{tabular}

voice and the different acoustic parameters, producing an alteration in the recognition of speaker's voices. Consequently, these changes may affect the SISS, in such a way that these systems may not recognize the user after a surgery in the vocal tract.

There are several studies that have assessed the influence of FESS on acoustic parameters or subjective changes in the patient's voice, ${ }^{4-9}$ but, to the author's knowledge, no work had previously studied the changes that FESS or other similar surgery may affect SISS.

In our study, basal presurgical GRBAS were significantly different between FESS patients and controls (Table 1). This difference could be due to the fact that FESS candidates suffered severe nasal obstruction and greater oral breathing, and this could alter the resonance of the vocal tract and therefore the perception of the voice and speech by a voice pathologist. After FESS, a significant change of GRBAS in the FESS group was found, while there were no significant changes in the control group (Table 2). One reason of this change may be the fact that by having a better nasal passage, the oral respiration decreases, and thus GRBAS score shows lower values in the following visits after surgery, while in the control group this does not happen.

On the other hand, there are several works reporting acoustic changes of voice after FESS. Kim YH et al in $2013^{7}$ studied 50 patients who underwent isolated FESS,

TABLE 4.

EER Score by Group and by Stage.

\begin{tabular}{lclc}
\hline GRUPO & EER1 & \multicolumn{1}{c}{ EER2 } & \multicolumn{1}{c}{ EER3 } \\
\hline Control & 0 & 3.84615385 & 3.84615385 \\
FESS & 0 & 3.7037037 & 14.8148148 \\
\hline
\end{tabular}

septoplasty, and the combination of both. They only observed an improvement in GRBAS, and an increase of nasalance at 1 month after surgery, but after 3 months these returned to presurgical values. Brandt et $\mathrm{al}^{8}$ elaborated a study of 14 patients operated on septoplasty, FESS and septorhinoplasty, in which recordings were made of the vowels held $/ \mathrm{a} /, / \mathrm{i} /$ and $/ \mathrm{u} /$ and reading text. They analyzed: F0, Jitter, Shimmer, HNR and conclude that they do not found changes in any parameter except in HNR. Acar et $\mathrm{al}^{9} \mathrm{col}^{\mathrm{c}}$ lected a total of 43 patients with polyposis and divided them according to their degree on the Lildholdt scale into three groups (grade I, II, and III) to analyze the acoustic parameters after surgery. Then, they recorded the vowel held /a/ and analyze the F0, Jitter, Shimmer, and NHR, preoperatively and at 6 weeks after FESS. They concluded that the results do not vary in the different groups except the Shimmer in group III, and there is a tendency to increase the F0, without being significant.

These three papers obtained nonconclusive results probably related to scarce and heterogeneous samples, without comparing them to a control group.

In our study, after FESS the acoustic parameters showed that F0 decreases 3 months after surgery, with statistical significance compared to the control group (Table 4). It is not known exactly why the F0 changed in the operated group, but in other published studies variations in the $\mathrm{F} 0$ have also been observed after FESS. ${ }^{20,21,22}$ According to the filter source theory, the F0 can be affected by modifying the vocal tract, as it occurs in this study. Therefore, modifications in the vocal tract may affect the source, explaining the effect in the F0, through a better nasal permeability in inspiration and a better resonance of the nasal cavity. Also, after FESS, nasal breathing improves because of a greater nasal passage, decreasing oral breathing in these patients. Another possibility is that this change in F0 can be associated to the 
changes in the subjective self-perception of the patient's voice after surgery, which was observed in half of our patients, correlating to the change in $\mathrm{F} 0{ }^{22}$

In relation to SISS, to our knowledge, this is the first article analyzing the behavior of these systems after surgery of the vocal tract of the speakers involved. In our study, we observed that FESS significantly increased the EER for speaker recognition with respect to the control group by a factor of 3.86 (14.81 versus 3.84 ).

These differences in the EER suggest that there are changes in the speech signal of some of the patients after surgery that affects the recognition systems leading to a wrong recognition of some of them. These results give innovative information applicable to the computer systems used nowadays.

Limitations of the study are that the use of rhinomanometry could have helped and granted more parameters to the study, it was not included in the protocol, as it had already been analyzed previously in the literature. ${ }^{6-9,21}$

In the same sense, it is not clear if the use of other vowels would have yielded different results as some works suggest. ${ }^{23}$ To this respect, further studies employing three repetitions of the rest of Spanish vowels must be addressed in the future. Also, the combination of traditional acoustic parameters and other new such as modulation spectrum morphological parameters ${ }^{24}$ or complexity measurements ${ }^{25}$ must be employed in future works.

Further studies including more types of surgery and patients in combination with other set-ups for speaker identification are needed to corroborate these results. This study can be the beginning of future research respecting that type of analysis in order to provide more information of the acoustic changes of the vocal tract after surgery, and their influence in the computer systems.

As a conclusion, in our study FESS produced changes in the vocal tract, with a decrease of F0, disturbing the perception of speech and affecting SISS performance, yielding lower speaker recognition rates 3 months after surgery in patients who undergone FESS.

\section{CONFLICT OF INTEREST}

None of the authors present a conflict of interests.

\section{SUPPLEMENTARY MATERIALS}

Supplementary material associated with this article can be found in the online version at https://doi.org/10.1016/j. jvoice.2019.02.012.

\section{REFERENCES}

1. Fokkens WJ, Lund VJ, Mullol J, et al. European Position Paper on Rhinosinusitis and Nasal Polyps 2012. Rhinol Suppl [Internet]. 2012:23:1-298.

2. Stammberger H. Functional Endoscopic Sinus Surgery: The Messerklinger Technique. Philadelphia, EEUU: Decker; 1991
3. Wigand ME. Transnasal ethmoidectomy under endoscopical control Rhinology. 1981;19:7-15.

4. Havel M, Kornes T, Weitzberg E, et al. Eliminating paranasal sinus resonance and its effects on acoustic properties of the nasal tract. Logoped Phoniatr Vocol. 2016:41:33-40.

5. Havel M, Ertl L, Bauer D, et al. Resonator properties of paranasal sinuses: preliminary results of an anatomical study. Rhinology. 2014;52:178-182.

6. Ungor C, Saridogan C, Yilmaz M, et al. An acoustical analysis of the effects of maxillary sinus augmentation on voice quality. Oral Surg Oral Med Oral Pathol Oral Radiol. 2013;115:175-184.

7. Kim YH, Lee SH, Park CW, et al. Nasalance change after sinonasal surgery: analysis of voice after septoturbinoplasty and endoscopic sinus surgery. Am J Rhinol Allergy. 2013:27:67-70

8. Brandt MG, Rotenberg BW, Moore CC, et al. Impact of nasal surgery on speech resonance. Ann Otol Rhinol Laryngol. 2014;123:564-570.

9. Acar A, Cayonu M, Ozman M, et al. Changes in acoustic parameters of voice after endoscopic sinus surgery in patients with nasal polyposis. Indian J Otolaryngol Head Neck Surg. 2014;66:381-385.

10. Beigi H. Speaker recognition: advancements and challenges. New Trends and Developments in Biometrics. InTech; 2012.

11. Aron. J. How innovative is Apple's new voice assistant, Siri? New Sci. $2011 ; 212: 24-29$.

12. Moro-Velázquez L, Gómez-García JA, Godino-Llorente JI, et al. Analysis of speaker recognition methodologies and the influence of kinetic changes to automatically detect Parkinson's Disease. Appl Soft Comput J. 2018;62:649-666.

13. Núñez Batalla F, Corte Santos P, Sequeiros Santiago G. Evaluación Perceptual de la Disfonía: Correlación con los Parámetros Acústicos y Fiabilidad. Acta Otorrinolaringol Esp. 2004;55:282-287.

14. Pereira ERBNBN, Tavares ELM, Martins RHG. Voice disorders in teachers: clinical, videolaryngoscopical, and vocal aspects. $J$ Voice. 2015;29:564-571

15. Godino-Llorente J, Osma-Ruiz V, Sáenz-Lechón N, et al. Acoustic analysis of voice using WPCVox: a comparative study with Multi Dimensional Voice Program. Eur Arch Oto-Rhino-Laryngology. 2008:265:465-476.

16. Mehta DD, Rudoy D, Wolfe PJ. Kalman-based autoregressive moving average modeling and inference for formant and antiformant tracking. $J$ Acoust Soc Am. 2012;132 17321746

17. Rajan P, Afanasyev A, Hautamäki V, et al. From single to multiple enrollment i-vectors: practical PLDA scoring variants for speaker verification. Digit Signal Process. 2014;31:93-101.

18. Povey D, Ghoshal A, Boulianne G, et al. The Kaldi speech recognition toolkit. IEEE 2011 workshop on automatic speech recognition and understanding. IEEE Signal Process Soc. 2011

19. Moreno A, Poch D, Bonafonte A, et al. Albayzin speech database: design of the phonetic corpus. Eurospeech 1993 Proc 3rd Eur Conf Speech Commun Technol. 1, 1993175-178.

20. Dehak N, Kenny PJ, Dehak R, et al. Front-end factor analysis for speaker verification. IEEE Trans Audio Speech Lang Process. 2011;19:788-798.

21. Koo SK, Kwon SB, Chon KM, et al. The role of the maxillary sinus on the voice. Eur Arch Otorhinolaryngol. 2015;272:2347-2350.

22. Kim SD, Park HJ, Kim GH, et al. Changes and recovery of voice quality after sinonasal surgery. Eur Arch Oto Rhino Laryngology. 2015;272: 2853-2859

23. Haque $\mathrm{S}$, Ali MH, Haque AKMF. Variability of acoustic features of hypernasality and it's assessment. Int J Adv Comput Sci Appl. 2016;7: 195-201.

24. Moro-Velázquez L, Gómez-García JA, Godino-Llorente JI, et al. Modulation spectra morphological parameters: a new method to assess voice pathologies according to the GRABS scale. BioMed Res Int 2015;1-13.

25. Arias-Londoño JD, Godino-Llorente J, Sáenz-Lechón N, et al. Automatic detection of pathological voices using complexity measures, noise parameters, and mel-cepstral coefficients. IEEE Trans Biomed Eng. 2011;58:370-379 